\title{
Smoke Detection Using GMM and Adaboost
}

\author{
Hyuntae Kim, Daehyun Ryu, and Jangsik Park, Member, IACSIT
}

\begin{abstract}
Detection performance of the smoke in the outdoor depends on weather conditions, the shadow by the movement of the sun, or illumination changes, etc. In this paper, a smoke detection system in conjunction with a robust background estimate algorithm to environment change in the outdoor in daytime is proposed. Gaussian Mixture Model (GMM) is applied as background estimation algorithm, and also, Adaboost algorithm is applied to detect the smoke for candidate region. Through the experiments with input videos obtained from a various weather conditions at the same actual road, the proposed algorithms were useful to detect smoke in the outdoor.
\end{abstract}

Index Terms-GMM, adaboost, smoke detection, outdoor environments.

\section{INTRODUCTION}

Video-based fire detection systems are generally of two types of methods. One is to use the color information of the flame and smoke, and the other one uses a dynamic movement [1], [2]. Erroneous detection occurs according to a similar color or lighting color in the surrounding environment.

In this paper, in order to reduce erroneous detection of smoke a method of detecting smoke using statistical characteristics of smoke proposed.

Gaussian Mixture Model is applied as background estimation algorithm, and Adaboost algorithm is applied to detect the smoke for candidate region in the proposed method.

\section{THE PROPOSED METHODS}

\section{A. Outline of the Proposed Algorithm}

When the system starts up, the first thing is to receive video input signal. Then, the image is converted to gray. The first frame or if less than the specified length of frame, the system will generate the GMM data and store background image. Next, the differential image is calculated by using the background image and input image. The candidate area is determined by morphological operations for a differential image. Using the Adaboost algorithm, it is determined whether the area of smoke finally.

Manuscript received December 5, 2013; revised January 15, 2014. This work (Grants No. C0116342) was supported by Business for Cooperative R\&D between Industry, Academy, and Research Institute funded Korea Small and Medium Business Administration in 2013. This research was also supported by Basic Science Research Program through the National Foundation of Korea(NRF) funded by the Ministry of Education, Science and Technology(2012M3C1A1048865)

Hyuntae Kim is with Department of Multimedia Engineering, Dongeui University, Busan, 614-714 South Korea (e-mail: htaekim@deu.ac.kr).

Daehyun Ryu is with Faculty of Information Technology, Hansei University, Kyunggi Province, South Korea (e-mail:dhryu@ @ansei.ac.kr).

Jangsik Park is with Department of Electronics Engineering, Kyungsung University, Busan, 608-736 South Korea (e-mail: jsipark@ks.ac.kr).

\section{B. GMM (Gaussian Mixture Model)}

Background subtraction is a particularly popular method for motion segmentation, especially under those situations with a relatively static background. The numerous approaches to this problem differ in the type of the background model and the procedure used to update the background model. Gaussian background models are among the most robust available [3]-[6]. (See Fig. 1).

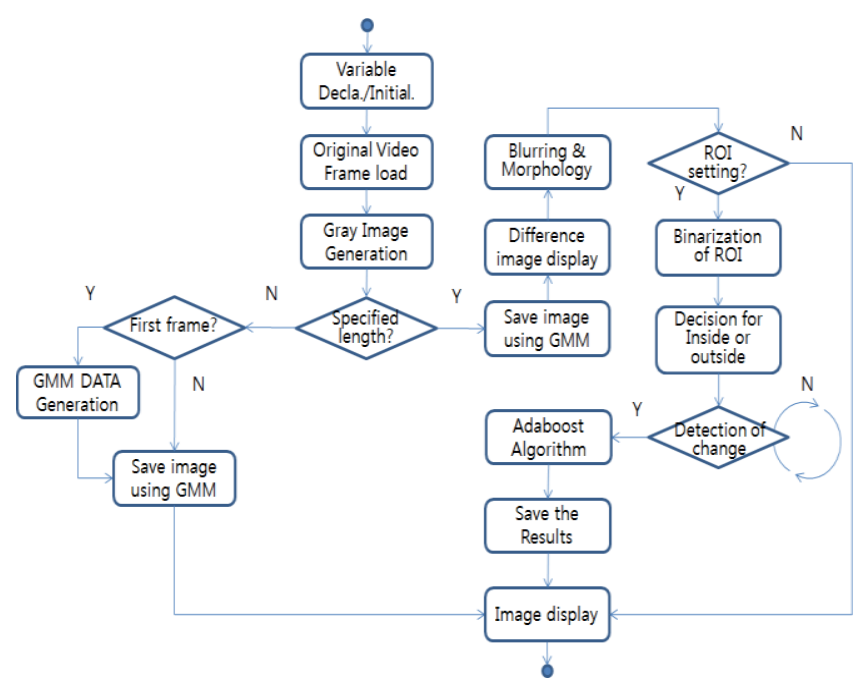

Fig. 1. The block diagram of the proposed system.

Each pixel in the scene is modeled by a mixture of $K$ Gaussian distributions. The probability that a certain pixel has intensity $x_{t}$ at time $\mathrm{t}$ is expressed as:

$$
P\left(x_{t}\right)=\sum_{i=1}^{K} \omega_{i} \times \eta\left(x_{t}, \mu_{i}, \sum_{i}\right)
$$

where $\omega_{i}$ is the weight, $\mu_{i}$ is the mean, $\sum_{i}=\sigma_{i}^{2}$ is the covariance for the $i^{\text {th }}$ distribution, and $\eta$ is a Gaussian probability density function:

$$
\eta\left(x_{t}, \mu_{i}, \sum_{i}\right)=\frac{1}{(2 \pi)^{\frac{1}{2}}\left|\sum\right|^{\frac{1}{2}}} e^{-\frac{1}{2}\left(x_{t}-\mu\right)^{T} \Sigma^{-1}\left(x_{t}-\mu\right)}
$$

The $K$ distributions are ordered based on $\omega_{i} / \sigma_{i}$ and the first $B$ distributions are used as a model of the background of the scene where $\mathrm{B}$ is estimated as:

$$
B=\arg \min \left(\sum_{i=1}^{b} \omega_{i}>T\right)
$$

The threshold $T$ is the minimum fraction of the background 
model. Background subtraction is performed by marking any pixel that is more than $\lambda(1.0 \sim 1.5$ in our experiments) standard deviation away from any of the $B$ distribution as a foreground pixel.

Thus, the candidate background region obtained through background estimation. Then Adaboost algorithm is used to detect smoke object.

\section{Adaboost}

This paper uses a total of 14 of the rectangular characteristics of the prototype. They belong to the edge, line, around the three types of abstract features. Viola given rectangle features than the prototype has been rotated 45 degrees to join the new features of the prototype rectangle. From characteristics of the prototype can be derived rectangular feature-rich, and methods are: the absence of rotating rectangular characteristics of the prototype, this can be vertically or horizontally to change the rectangle's side long; 45-degree angle for the rotation of rectangular features of the prototype, this can be along the positive (negative) 45-degree angle to change the rectangle side length [7], [8].

In order to rapidly calculate Haar-features, "Integral Image" method was used. Point $(x, y)$ integral as defined equation (4) as follow:

$$
i i(x, y)=\sum_{x^{\prime} \leq x, y^{\prime} \leq y} i\left(x^{\prime}, y^{\prime}\right)
$$

where $i i(x, y)$ is the integral image, $i(x, y)$ is the original image. Each point of the integral image is based on the physical meaning of the image with the point of origin for the diagonal ends of the pixel values within the rectangle end.

Set the initial conditions $i(-1, y)=i i(x,-1)=i i(-1,-1)=0$, using an iterative process can quickly calculate the corresponding image points in the value of the integral image that

$$
i i(x, y)=i i(x, y-1)+i i(x-1, y)-i i(x-1, y-1)+i(x, y)
$$

Points images, the images in any area within the pixel and can be by-point the value of the integral image obtained by simple addition and subtraction. For example, Fig. 2, from the ABCD four endpoints constitute a black rectangular area, its internal pixel value and can be A, B, C, D 4 endpoints of the integral image value through the simple addition and subtraction method to be, that is,

$$
\operatorname{Sum}_{\mathrm{Re} g i o n}=i i(D)+i i(A)-i i(B)-i i(C)
$$

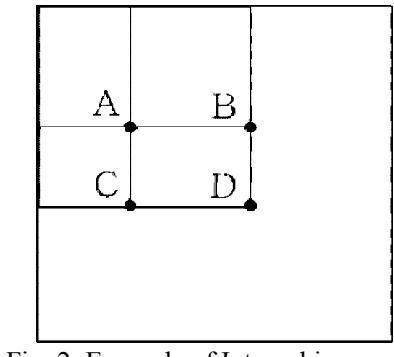

Fig. 2. Example of Integral image.

This just a simple addition of the fast algorithm for computing the feature extraction step greatly reduced the computational complexity to ensure that the algorithm is able to adapt to real-time system requirements.

The process of data training is:

1) Input: Training examples $\left(x_{i}, y_{i}\right), i=1,2, \ldots, N$ with positive $\left(y_{i}=1\right)$ and negative $\left(y_{i}=0\right)$ examples.

2) Initialization: weights $w_{1, i}=\frac{1}{2 m}, \frac{1}{2 l}$ with $m$ negative and $l$ positive examples.

3) For $t=1,2, \ldots, T$ :

- Normalize all weights $w_{t, i} \leftarrow \frac{w_{t, i}}{\sum_{j=1}^{n} w_{t, j}}$ so that $w_{t}$ is a probability distribution.

ü For each feature $j$ train classifier $h_{j}$ which is restricted to using a single feature. The error is evaluated with respect to $w_{t}, \varepsilon_{j}=\sum_{i} w_{t, i} \mid h_{j}\left(x_{i}-y_{i}\right){ }^{*}$

ü Choose $h_{t}$ with lowest error $\varepsilon_{t}$.

ü Update weights: $w_{t+1, i}=w_{t, i} \beta_{t}^{1-e_{i}}$ with $e_{i}=\left\{\begin{array}{cc}0 & x_{i} \text { correctlyclassified } \\ 1 & \text { otherwise }\end{array}\right.$ and $\beta_{t}=\frac{\varepsilon_{t}}{1-\varepsilon_{t}}$.

4) Final strong classifier:

$$
h(x)=\left\{\begin{array}{ccc}
1 & : & \sum_{t=1}^{T} \alpha_{t} h_{t}(x) \geq 0.5 \sum_{t=1}^{T} \alpha_{t} \quad \text { with } \alpha_{t}=\log \left(\frac{1}{\beta_{t}}\right) \\
0 & : & \text { otherwise }
\end{array}\right.
$$

\section{EXPERIMENTAL RESULTS}

The experiments were performed using YouTube video, $320 \times 240$ at 25 frames per second. Window $7 \mathrm{PC}$, Intel(R) Core(TM) i5-2500 CPU @ 3.30GHz, Visual Studio 2010, and public library OpenCV were used.

Experimental results for smoke, as shown in Fig. 3 and Fig. 4 are shown in accordance with the progress of the smoke. As shown in Fig. 3, even outdoors was confirmed that the detection of smoke well. And Fig. 4 shows the performance of the proposed system where the smoke and other moving objects were present in a situation. In order to distinguish between them, the red box is the area with smoke and non-smoke objects set as a green box.

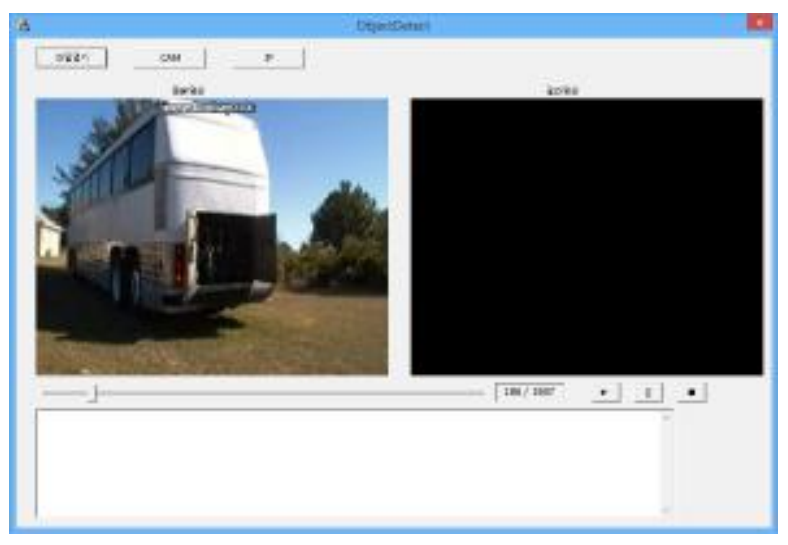

(a) Before smoke. 


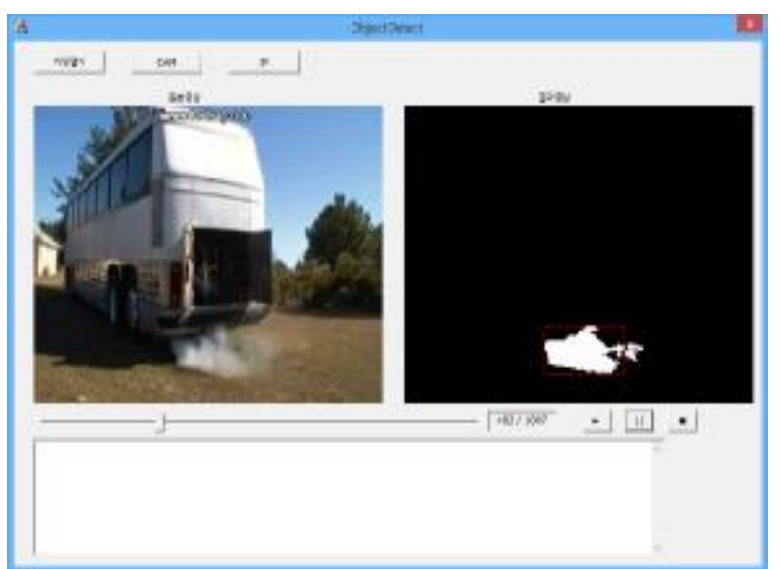

(b) Smoke generation early.

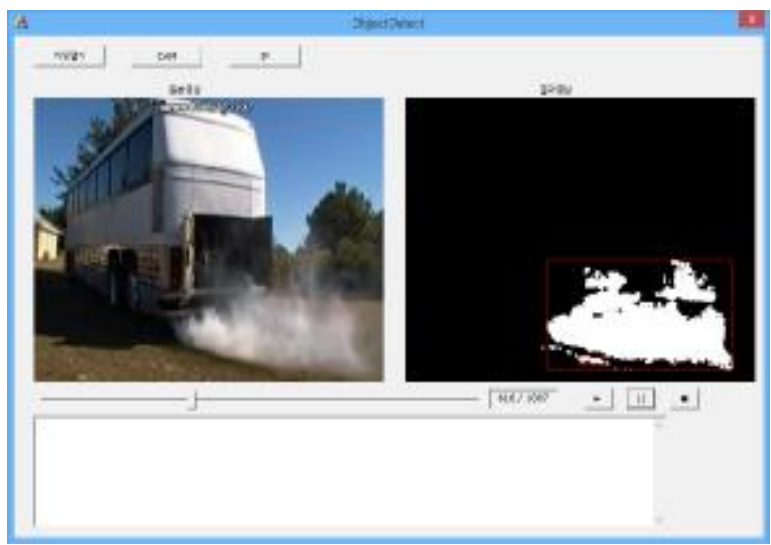

(c) Smoke spread.

Fig. 3. Experimental results for outdoor smoke video.

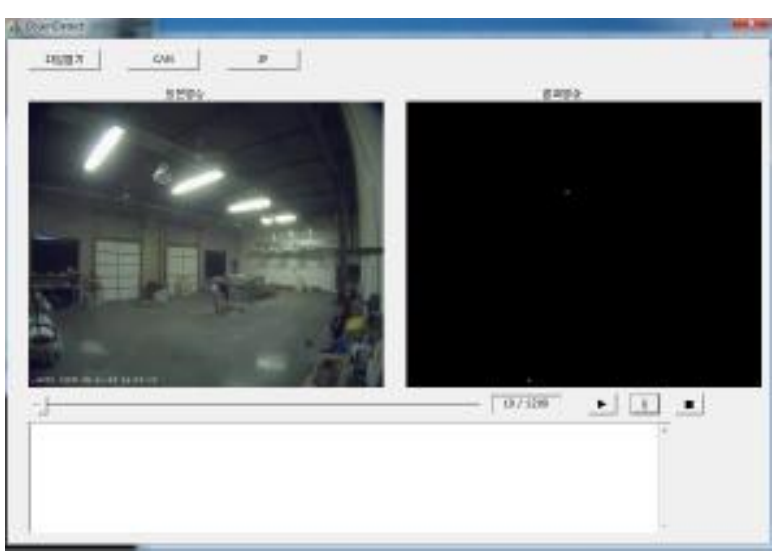

(a) Before smoke.

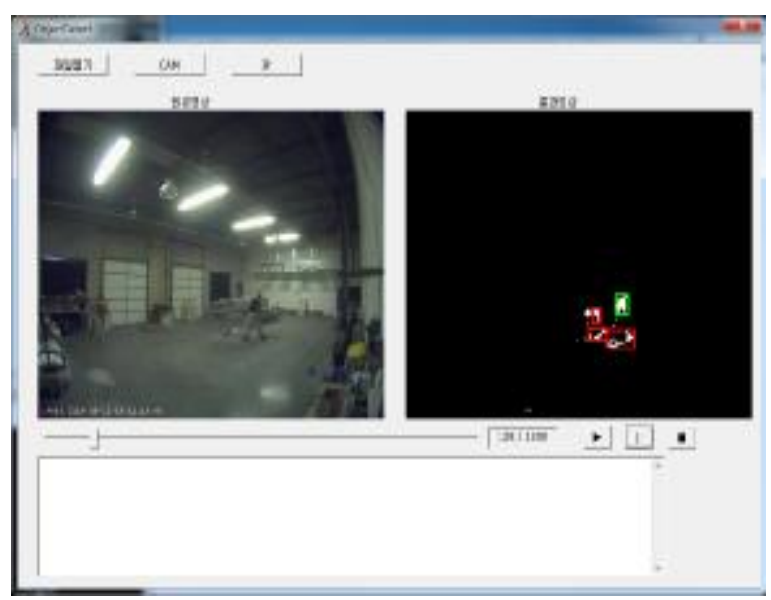

(b) Smoke generation early.

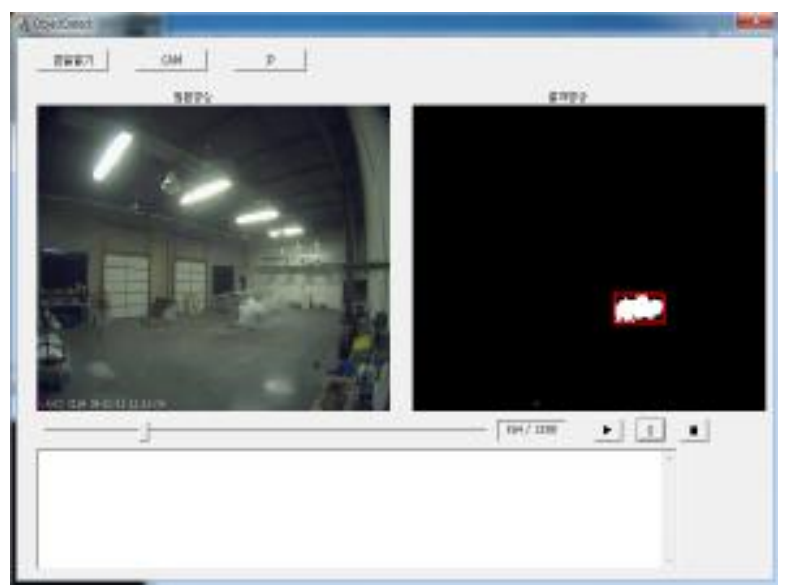

(c) Smoke spread.

Fig. 4. Experimental results for the situation with smoke and non-smoke.

\section{CONCLUSION}

As the experimental results using outdoor video signal, the proposed background estimation and smoke object detection methods was effective in detecting outdoor smoke.

\section{REFERENCES}

[1] B. U. Toreyin, Y. Dedeoglu, U. Gudukbay, and A. E. Cetin, "Computer vision based method for real-time and flame detection," Pattern Recognition Letters, Elsevier, 2005.

[2] G. Healey, D. Slater, T. Lin, B. Drda, and A. D. Goedeke, "A system for real-time fire detection," in Proc. IEEE Computer Vision and Pattern Recognition Conference, 1993, pp. 605-606.

[3] D. Zhou and H. Zhang, "Modified GMM background modeling and optical flow for detection of moving objects," in Proc. IEEE Systems, Man and Cybernetics, 2005.

[4] K. Gyuyeong, K. Hyuntae, P. Jang-Sik, and Y. Yunsik, "Vehicle tracking based on kalman filter in tunnel," Springer ISA 2011, CCIS vol. 200, pp. 250-256. 2011.

[5] K. Hyuntae, L. Geunhoo, P. Jang-Sik, and Y. Yunsik, "Vehicle detection in tunnel using Gaussian mixture model and mathematical morphological processing," The Journal of the Korea Institute of Electronic Communication Science, vol. 7, no. 5, pp. 967-974, 2012.

[6] P. Keunsoo and K. Hyuntae, "A study for video-based vehicle surveillance on outdoor road," The Journal of the Korea Institute of Electronic Communication Science, vol. 8, no. 11, pp. 1647-1653, 2013.

[7] P. Deng, "Multi-profile face detection method based on color and AdaBoost," Beijing Institute of Technology, Department of Biomedical Engineering, 2008.

[8] Z. Sun, G. Bebis, and R. Miller, "On-road vehicle detection using evolutionary Gabor filter optimization," IEEE Trans. Intelligent Transportation Systems, vol. 6, no. 2, pp. 125-137, 2005.

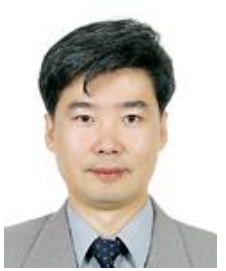

Hyuntae Kim was born in 1966 in Busan, Republic of Korea. He received the B.S., the M.S. and the Ph.D. degrees in the electronics eng. from Pusan National University, Korea in 1989, 1995 and 2000, respectively. He joined the Dongeui University in Korea as a professor in the Multimedia Engineering Department since March 2002. He was a visiting professor at Georgia Institute of Tech. in USA at 2008. 


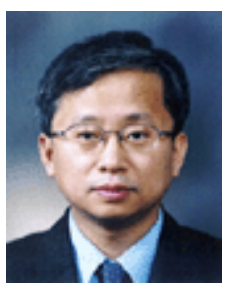

Daehyn Ryu received the B.S., the M.S. and the $\mathrm{Ph} . \mathrm{D}$. degrees in the electronics eng. from Pusan National University, Korea in 1983, 1985 and 1997, respectively. He was a researcher at the ETRI from 1987 to 1998 . He joined the Hansei University in Korea as a professor in the Electronics Engineering Department since March 1998.

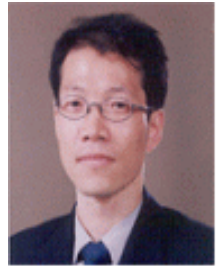

Jangsik Park was born in 1965 in Busan, Republic of Korea. He received the B.S., the M.S. and the Ph.D. degrees in the Electronics Eng. from Pusan National University, Korea in 1992, 1994 and 1999, respectively. He joined the Kyungsung University in Korea as professor in the Electronics Engineering Department since March 2011. 